\title{
ERUPTIVE PROMINENCE ASSOCIATED WITH LIMB FLARE OF 25 JANUARY 1991
}

\author{
WAHAB UDDIN and V. K. VERMA* \\ Uttar Pradesh State Observatory, Manora Peak, \\ Naini Tal-263 129, India
}

December 1, 1996

\begin{abstract}
We have observed an eruptive prominence at the east solar limb on 25 January 1991 which started earlier than 0623 UT and was associated with a limb flare (S16 E90) of class 1B/ X10.0. We have recorded a huge mass ejection in the corona by the limb flare associated eruptive prominence. The eruptive prominence ejected a part of the loop in the corona with maximum speed of about $1280 \mathrm{~km} / \mathrm{sec}$. The ejected material attain height upto $150,000 \mathrm{~km}$ in the corona and finally faded/disappeared in the corona. During the ascending phase of the prominence material in the corona there was a unscrewing of the loop system associated with the eruptive prominence. The type II, III, and IV radio bursts were also reported by a number of Radio Observatories during observation of the eruptive prominence. The high flux of sudden ionospheric disturbances and the solar radio emissions on fixed frequencies $(245-80000 \mathrm{MHz})$ were also recorded. The eruptive prominence associated with limb flare also shows increased proton flux $(>10 \mathrm{MeV})$ during its occurence. The flare was classified as X10.0 flare. In this paper we have analysed the observed data and compared it with the theoretical model of the solar flare.
\end{abstract}

Key words: Limb flare - Eruptive prominence - mass ejections

\section{Introduction}

It is well known that there are some flare-associated phenomena which are related to mass motions or ejections following or preceding the flare. Unfortunately, in most of the observatories, where flares are observed in the light of $H_{\alpha}$ line, the observations of these phenomena are not possible on the disk. But if the flare occurs on the limb and it is accompanied by an associated phenomenon, it can be observed in detail. Their detection and morphological development are valuable for theoretical studies as well as from an observational point of view, because occurrence of such phenomena on the limb are rare. Recent observations indicate that global instability of the magnetic field configuration in the active region drives both the filament eruption and the impulsive phase energy release which is manifested in hard X-ray emission (Kahler et. al., 1988). The impulsive phase energy release is generally agreed to arise from magnetic field annihilation and reconnection, which may occur in a current sheet beneath the erupting filament, perhaps at a

* On leave from his original Institute 
juncture with newly emergent flux tubes, or with portions of the erupting filament itself (Kopp and Pneuman, 1976; Heyvaerts, Priest and Rust, 1977). It was shown by Kaastra (1985) that as the filament moves upwards, a neutral line is formed beneath it, which becomes the site of particle acceleration during the flare, because of the electric field induced by the motion of the filament current (Martens, 1988, Martens and Kuin, 1989). The acclerated particles, as soon as they leave the sheet, precipitate into the chromosphere along the field lines and are responsible for the generation of hard X-rays at the impact site, as well as $H_{\alpha}$ emission. The particle beams further cause chromospheric evaporation which leads to the observed coronal EUV-and soft X-ray emission (Canfield et al., 1983).

In this paper, we present study of eruptive filament with limb flare of $1 \mathrm{~B} / \mathrm{X} 10$ associated with long duration microwave, type II, type III and type IV radio bursts and proton emission $(>10 \mathrm{MeV})$. A possible explanation for these emissions are also given.

\section{Observational Data}

Observations of eruptive filament with limb flare in $\mathrm{H} \alpha$ were made with the $15 \mathrm{~cm}$ coude telescope at UPSO, Naini Tal. The event occured on 1991 January 25 in active region NOAA 6471 at location (S16 E90). When the observations were started the filament eruption was in its early phase and it attained a loop shape structure with a bright blob of huge plasma above the limb at 063242 UT fast sequence of filament eruption were taken. Filament eruption show very dynamic behaviour (Fig. 1). It seems that there is a low brightness at the bottom of one leg in the early phase. When the eruptive filament attains question mark shape (?) i.e. with one leg having disappeared and one leg is detached slightly around 063641 there is considerable brightness at the limb where the filament leg is anchored. This was the start. Later, the limb flare became very bright and it appeared as a oval shaped structure projected above the limb (Fig. le and 1f). Enhancements of emission in other wavelengths were also observed from the above event. Soft X-ray, microwave burst, radio burst, SIDs and proton emission data are available for correlation study. Solar Geophysical Data (1991) of limb flare.

\section{Data Analysis}

The $\mathrm{H} \alpha$ observations of the eruptive prominence which is associated with limb flare are very useful for the morphological study in the optical region. When the observations were started the prominence already became active 
and it started rising with a very high speed of about $1300 \mathrm{~km} / \mathrm{s}$. A sequence of $\mathrm{H} \alpha$ images of an erupting prominence with a limb flare is shown in Figure 1. It appears that the limb flare began under the filament and filament eruption is a precurser for the underlying flare. In the beginning flare was not visible at footpoints of the erupting prominence around 063242 UT. After 4 min flare seems to appear at the foot point when the prominence attains its maximum height.

The correlation study of $\mathrm{H} \alpha$ observations with other emissions in different wavelength(not shown here) we found that type III radio bursts at 06 31 and a number of microwave long duration great bursts observed in the interval from $0616-0652$ at different phases of the event. At 0631 UT one very intense (peak flux $57000 \times 10^{-22} \mathrm{w} / \mathrm{m}^{2} \mathrm{~Hz}$ ) burst at $245 \mathrm{MHz}$ and type III radio burst occurred when the filament eruption initiated. We have plotted (not shown here) peak flux of microwave burst versus frequency of the microwave bursts observed at radio observatories which show two peaks, indicating two emissions sources. Soft X-ray observations from GOES satellite show high peak with sharp rise and slow decline from $0630 \mathrm{UT}$ to 10 $00 \mathrm{UT}$ with peak at $0635 \mathrm{UT}$ and it falls in X-10 class of X-ray flare (SGD 1991). Proton emission enhancement $(\geq 10 \mathrm{MeV})$ also observed which is associated with the above event. A long duration SID from 0620 to 1106 UT also occurred as a result of the very dynamic event.

\section{Results and Discussion}

To summarize the characteristics of the eruptive prominence and associated limb flare we have $H_{\alpha}$ observations and the observations in other frequencies are avaliable in SGD 1991. We found that the eruptive prominence is very dynamic and has a twisted structure which is clearly visible in the legs of the loop. The separation of legs at the footpoints is considerably small and the flare ribbons formed at the bottom of the legs and it seems a two ribbon flare because due to forshortening effect the ribbons are not separately visible. Such type of errupting prominences are usually found to be associated with two ribbon flares and they also show very dynamic nature. It seems the energy release took place in two steps: (i) At the time when the filament took place and (ii) The other phase when the limb flare started. These two steps are very important phases in these steps a bulk energization took place and these energetic electrons generated type II, III, IV radio bursts which travelled into the interplanetary medium. From the above results it seems that such type of events are very energetic in coronal region i.e. at high temperature region and may be associated with coronal mass ejection (CME). We feel that the whole story of the event observed by us can be explained very well with the help of circuit model given by Martens and Kuin(1989). 


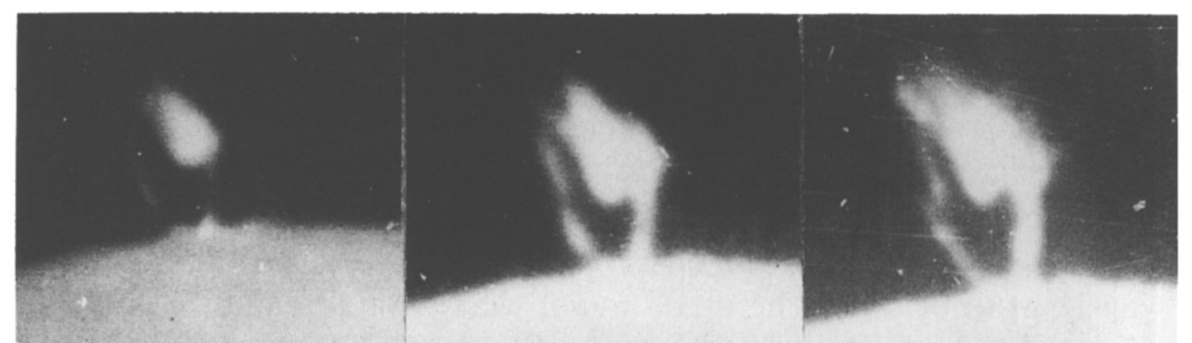
(a) 063242 UT
(b) 063322 UT
(c) $063432 \mathrm{UT}$

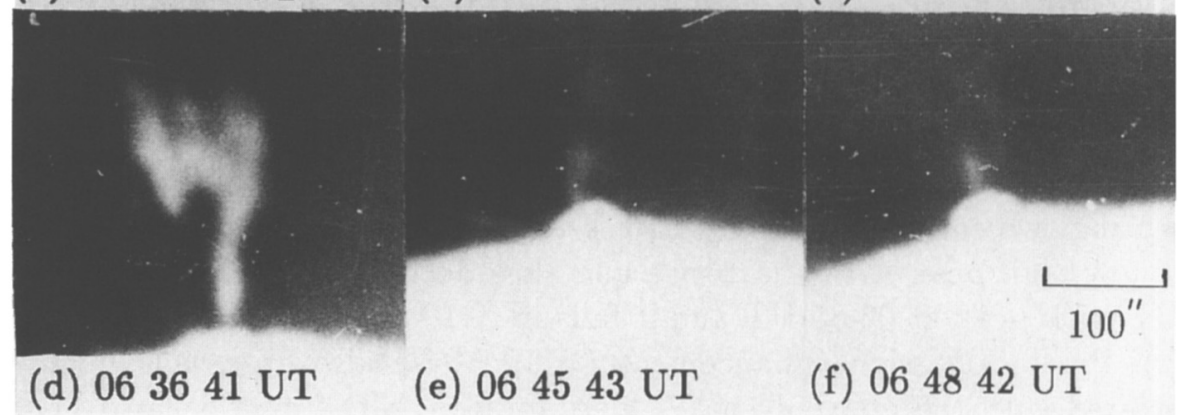

Fig. 1. Selected $H-\alpha$ fitergrams of the ereptive prominence with limb flare on 25 January 1991.

The detailed internal structure of the filament with its individual flux tubes is ignored in the model. Such type of events can also be understood well on the basis of the model given by Sturrock (1986).

We now conclude that a large filament associated with limb flare is ejected out from the sun into interplanetary space. Because its speed is very high $\approx 1300 \mathrm{kms}^{-1}$ so it produced a bow shock. Such a shock would tend to maintain its strength as it propagates, whereas the strength of the blast wave tends to decrease rapidly as it propagates. In this case, the expansion of the magnetic field system will weaken the magnetic trap, so that particles can escape into interplanetary space. The filament eruption is so dynamic its seem that it is a spray. The emission in different wavelengths for long durations associated with the event shows that this is a very energetic and complex event and such type of events may be associated with CME.

\section{References}

Canfield, R. C., Gunkler, T. A., Hudson, H. S., Acton, L. W. Leibacher , J. W. and Kiplinger, A. L. : 1983, Adv. Space Res. 2, 145.

Farnik, F., Kaastra, J. S., Kalman, R., Karlicky, M., Slottge, C. and Valnicek, B.: 1983, Solar Phys. 89, 355. 
Heyvaerts, J., Priest, E. R. and Rust, D. M.: 1977, Astrophys. J. 216, 123.

Kaastra, J. S. : 1985, 'Solar Flares:An electrodynamical Model', Thesis, University of Utrecht.

Kahler, S. W., Moore, R. L., Kane, S. R. and Zirin, H. : (1988), Astrophys. J. 328, 824.

Kopp, R. A. and Pneuman, G. W. : (1976), Solar Phys. 50, 85.

Martens, P. C. H. : 1988, Astrophys. J. 330, L 131.

Martens, P. C. H. and Kuin, N. P. M. : 1989, Solar Phys. 122, 263.

Solar Geophysical Data 1991, Part I, No. 559 and Part II No. 563 U. S. Department of Commerce Boulder Colorado, USA.

Solar Geophysical Data (Preliminary Report and Forecast) 29 Janurary 1993 NOAA, Boulder USA.

Sturrock, P. A. : 1986 in P. Simon (ed.), Proc. Solar Terrestrial Prediction Workshop, (France: Meudon Obs.), (in press). 